\title{
Research on Thermal Multivariable System Identification Based on Living Operating Data
}

\author{
Shihe $\mathrm{CHEN}^{1}, \mathrm{Xi}_{\mathrm{ZHANG}}{ }^{1}$, Jianguo DUAN ${ }^{2}$, Li ZHANG ${ }^{2}$, Xichun BAO ${ }^{2}$, \\ Yuguang $\mathrm{NIU}^{2}$, Zhongwei LIN ${ }^{2}$ \\ ${ }^{1}$ Electric Power Research Institute of Guangdong Power Grid Corporation, Guangzhou, 510000, \\ China \\ ${ }^{2}$ School of control and computer engineering, North China Electric power University, Beijing, \\ 102206, China \\ email: duanjianguo@ncepu.edu.cn
}

\begin{abstract}
Keywords: Thermal Power Plant; Multi-variable Loop; Closed-loop Identification; Principle Component Analysis; Subspace Algorithm
\end{abstract}

\begin{abstract}
This paper introduces an identification algorithm for a thermal system with multi-variable closed-loops. First, a kind of subspace identification algorithm based on the principle component analysis (PCA) is analyzed. Next, the PCA is used to identify the parameters of the process, where dynamic model of coordinated control system (CCS) of power plant is obtained. Finally, an example for a subcritical drum boiler is used to illustrate the effectiveness of the introduced identification algorithm. The results demonstrate the contributions of the developed identification method to the closed-loops in thermal power plant
\end{abstract}

\section{Introduction}

Modern thermal power plant, which is large inertia, nonlinear, and strong coupled, is a typical multi-input multi-output (MIMO) object [1]. The CCS is the most important control system of power plant, and is regarded as one of the most complex control system in the thermal power plant. For the strong coupling and time-varying, the CCS is very difficult to obtain a desired performance with classical PID controller. Furthermore, with the large-scale wind power integrated into the grid, recent grid codes have specified higher requirements of thermal power plants.

The advanced control theories may achieve better performance than conventional PID controllers used. However, those advanced control theories usually need the high accurate model to maintain the desired performance. However, industrial processes are general nonlinear and multivariable systems, which are not easy to identify the accurate parameters by using conventional identification methods, such as least square identification (LSI).

In recent years, the subspace model identification (SMI) is widely used to identify multivariable systems. SMI has a better numerical reliability and a modest computational complexity compared with the prediction error method (PEM), particularly when the number of outputs and states is large [2-5].SMI based multivariable output error state space (MOESP) has been proposed [6]. Ljung and McKelvey extended the subspace identification based on autoregressive exogenous (ARX) model into LS problems [7]; Van Overschee and De Moor provided a generic method for closed-loop subspace identifications [8].For error-in-variable (EIV) model structure, Huang et al. proposed a subspace identification method based on orthogonal projection and instrumental variables [9]. Aiming at solving the open-loop error in variable (EIV) identification problem, Wang and Qin [10] developed an instrument variable subspace identification method via PCA, where the bias has been delivered into the closed-loop identification.

This paper introduces a PCA based SMI method, where PCA is used to identify parameters of the state-space equation of the process by EIV formulation. A simulation example for a thermal power plant is given to illustrate the performance of the identification method. The results show that the proposed SMI method provides a more accurate identification in comparison with LSI used. The paper is organized as follows. The subspace identification method based on PCA is presented in 
Section II. In Section III, a simulation example provides to demonstrate the contributions. Finally, conclusions are drawn in Section IV.

\section{SMI with PCA Approach}

A typical SMI algorithm contains two steps: $H^{d}$;

1. Identification of the extended observability matrix $\Gamma_{f}$ and a block triangular Toeplitz matrix

2. Estimation of the system matrices A, B, C and D from the identified observability matrix and the Toeplitz matrix.

In this section we present an EIV SMI algorithm based on PCA, denoted as SMI-PCA. The following is the discrete-time linear time-invariant (LTI) state space model to represent a controlled object to be identified,

$$
\begin{aligned}
& x(k+1)=A x(k)+B u(k)+K e(k) \\
& y(k)=C x(k)+D u(k)+e(k)
\end{aligned}
$$

Here $u(k) \in R^{m \times 1}, y(k) \in R^{l \times 1}$ and $x(k) \in R^{n \times 1}$ are input, output and state variables, respectively. $e(k) \in R^{l \times 1}$ is the zero mean white noise. $K$ is the Kalman filter gain.

In order to describe the system dynamics, we use the extended state-space model. For an arbitrary time point $k$ taken as the current time, we define the past and future output vectors and the Hankel output matrices as the following:

$$
\begin{aligned}
& u_{p}(k)=\left[\begin{array}{llll}
u(k-p) & u(k-p+1) & \cdots & u(k-1)
\end{array}\right]^{\mathrm{T}} \in R^{l p} \\
& u_{f}(k)=\left[\begin{array}{llll}
u(k) & u(k+1) & \cdots & u(k+f-1)
\end{array}\right]^{\mathrm{T}} \in R^{l f} \\
& U_{p}=\left[\begin{array}{llll}
u_{p}(k) & u_{p}(k+1) & \cdots & u_{p}(k+N-1)
\end{array}\right] \in R^{m p \times n} \\
& U_{f}=\left[\begin{array}{llll}
u_{f}(k) & u_{f}(k+1) & \cdots & u_{f}(k+N-1)
\end{array}\right] \in R^{m f \times n}
\end{aligned}
$$

Where subscript $p$ and $f$ stand for the past and future, and $p \geq f>n$. The vectors $y_{p}(k)$ and $y_{f}(k)$ are similarly to the $u_{p}(k)$ and $u_{f}(k)$, respectively. By iterating Eqs. (1), we use the Hankel data matrix instead of the data vector, the extended model is rewritten in the following form [10]:

$Y_{f}=\Gamma_{f} X_{f}+H_{f}^{d} U_{f}+H_{f}^{s} E_{f}$

Where $Y_{f}$ and $E_{f}$ have the same structure as the $U_{f}$, and

$$
\Gamma_{f}=\left[\begin{array}{c}
C \\
C A \\
\vdots \\
C A^{f-1}
\end{array}\right] \in R^{m f \times n}
$$

is the extended observability matrix with rank $n$. The following matrixes

$$
H_{f}^{d}=\left[\begin{array}{cccc}
D & 0 & \cdots & 0 \\
C B & D & \cdots & 0 \\
\vdots & \vdots & \ddots & \vdots \\
C A^{f-2} B & C A^{f-3} B & \cdots & D
\end{array}\right] \in R^{m f \times l f}
$$

and

$$
H_{f}^{s}=\left[\begin{array}{cccc}
0 & 0 & \cdots & 0 \\
C & 0 & \cdots & 0 \\
\vdots & \vdots & \ddots & \vdots \\
C A^{f-2} & C A^{f-3} & \cdots & 0
\end{array}\right] \in R^{m f \times n f}
$$

are two block Toeplitz matrices. 
Considering (6), for closed-loop identification, the future disturbance $E_{f}$ is no longer independent of the future input $U_{f}$ due to the feedback.

To solve this problem, by adopting the EIV structure of Wang and Qin [10], we move the term related to $U_{f}$ into the left hand side of Eq. (6) as it would be a troublesome term if left in the right hand side of the equation. Substituting (7) into (10),

$$
\left[\begin{array}{ll}
I & -H_{f}^{d}
\end{array}\right]\left[\begin{array}{c}
Y_{f} \\
U_{f}
\end{array}\right]=\Gamma_{f} X_{f}+H_{f}^{s} E_{f}
$$

Using the short-hand notation

$$
W_{f}=\left[\begin{array}{ll}
Y_{f} & U_{f}
\end{array}\right]^{\mathrm{T}}
$$

Eq. (10) can be simplified as

$$
\left[\begin{array}{ll}
I & -H_{f}^{d}
\end{array}\right] W_{f}=\Gamma_{f} X_{f}+H_{f}^{s} E_{f}
$$

Performing an orthogonal projection of Eq. (12) onto the row space of $W_{p}$ yields

$$
\left[\begin{array}{ll}
I & -H_{f}^{d}
\end{array}\right] W_{f} / W_{p}=\Gamma_{f} X_{f} / W_{p}+H_{f}^{s} E_{f} / W_{p}
$$

Where $W_{p}=\left[\begin{array}{ll}Y_{p} & U_{p}\end{array}\right]^{\mathrm{T}}$

The last term of Eq. (13) is an orthogonal projection of the future disturbance $E_{f}$ onto the row space of past input and output matrix $W_{p}$, which is zero. Namely,

$$
H_{f}^{s} E_{f} / W_{p}=0
$$

Therefore, Eq. (13) can be simplified to

$$
\left[\begin{array}{ll}
I & -H_{f}^{d}
\end{array}\right] W_{f} / W_{p}=\Gamma_{f} X_{f} / W_{p}=\Gamma_{f} \hat{X}_{f}
$$

Where $\hat{X}_{f}=X_{f} / W_{f}$, and $\hat{X}_{f}$ is the Kalman filter state. The orthogonal projection of Eq. (13) onto the row space of $W_{p}$ results in Eq. (15), which includes a multiplication term between the extended observability matrix Ci and non-steady state Kalman state $\hat{X}_{f}$.

Denoting $\Gamma_{f}{ }^{\perp}$ as the orthogonal complement of $\Gamma_{f}$ with full column rank, multiplying both sides of Eq. (15) by $\Gamma_{f}{ }^{\perp}$, Eq. (15) can be transformed to

$$
\left[\Gamma_{f}{ }^{\perp}\right]^{\mathrm{T}}\left[\begin{array}{ll}
I & -H_{f}^{d}
\end{array}\right] W_{f} / W_{p}=0
$$

Denoting $Z=W_{f} / W_{p}$, the problem is transferred to find the orthogonal column space of $Z$, which should equal to the column space of $\left(\left[\Gamma_{f}^{\perp}\right]^{\mathrm{T}}\left[\begin{array}{ll}I & -H_{f}^{d}\end{array}\right]\right)^{\mathrm{T}}$.

Perform SVD decomposition of $Z$ as

$$
Z=\left(\begin{array}{ll}
U_{1} & U_{2}
\end{array}\right)\left(\begin{array}{ll}
\Sigma & 0 \\
0 & 0
\end{array}\right)\left(\begin{array}{c}
V_{1}^{\mathrm{T}} \\
V_{2}^{\mathrm{T}}
\end{array}\right)
$$

With Eq. (28), one can easily find the orthogonal column space of $\mathrm{Z}$, which is $U_{2}$. Therefore

$$
\left(\left[\Gamma_{f}^{+}\right]^{\mathrm{T}}\left[\begin{array}{ll}
I & -H_{f}^{d}
\end{array}\right]\right)^{\mathrm{T}}=U_{2} M
$$

Where $M$ is any constant nonsingular matrix and is typically chosen as an identity matrix [11]. Partition

$$
U_{2} M=\left(\begin{array}{l}
P_{1} \\
P_{2}
\end{array}\right)
$$

Then Eq. (29) can be written as 


$$
\left(\begin{array}{c}
\Gamma_{f}^{\perp} \\
-\left(H_{f}^{d}\right)^{T} \Gamma_{f}^{\perp}
\end{array}\right)=\left(\begin{array}{l}
P_{1} \\
P_{2}
\end{array}\right)
$$

Therefore,

$$
\begin{aligned}
& \Gamma_{f}{ }^{\perp}=P_{1} \\
& -\left(H_{f}^{d}\right)^{T} \Gamma_{f}{ }^{\perp}=P_{2}
\end{aligned}
$$

The remaining problem is to solve for $\Gamma_{f}$ and $H_{f}^{d}$, and then to extract the system matrices $A$, $B, C, D$ from $\Gamma_{f}$ and $H_{f}^{d}$. We refer to [10] for a discussion on the detailed solution procedure.

\section{Dynamic Simulations}

In Fig. 1 is the low-order nonlinear model of unit [12][13]. On the one hand, the model is a reflection of the energy balance between the systems, on the other hand, it reflects essential nonlinearity features of the system. Most of research and analysis of CCS of is based on this model in China, where $\mu_{B}, \mu_{T}, N_{E}$ and $P_{T}$ denote fuel command, opening of the main steam valve, output active power, and throttle pressure.

Taking into account the small perturbations characteristics of CCS at rated operating conditions, drum boiler-turbine units can be simplified to the dual-input dual-output dynamic linear model displayed in Fig. 2.

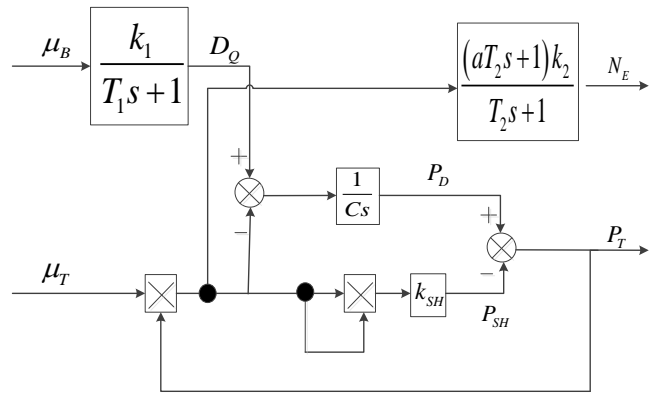

Fig. 1 Simplified nonlinear model of CCS

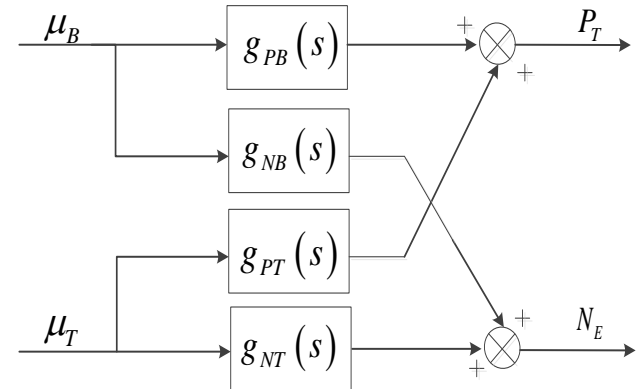

Fig.2 Linearized model of CCS

The relationship between control and process output variables can be expressed as a transfer function as follows:

$$
\left[\begin{array}{c}
P_{T} \\
N_{E}
\end{array}\right]=\left[\begin{array}{ll}
g_{P B}(s) & g_{P T}(s) \\
g_{N B}(s) & g_{N T}(s)
\end{array}\right] \times\left[\begin{array}{l}
\mu_{B} \\
\mu_{T}
\end{array}\right]=\mathrm{G}(s)\left[\begin{array}{c}
\mu_{B} \\
\mu_{T}
\end{array}\right]
$$

\section{A. Model Identification}

In this paper, the model of the CCS of 600MW power plant is employed as the identification object. Data of identification is selected from the living operating records, where the operating point is at the $60 \%$ rated capacity of the power unit. Under the chosen operating point, $\mu_{B}$ and $\mu_{T}$ are the input signals for the identification, while, $N_{E}$ and $P_{T}$ are the identified outputs. The filtrated 1400 pairs of the data, of which sampling period is $1 \mathrm{~s}$, are used to identify the parameters of the linearized state space model. Based on the obtained model, controlled object can be identified.

Next, we use the Akaike Information Criterion (AIC) to identify the order of the model, which was originally proposed by Akaike [14] and extended by Larimore for SMI[15][16]. The order of controlled object can be obtained from the AIC index based on characteristic polynomial (CP) method [10]. The AIC based on CP indicates that the system order is two shown in Fig. 3. Then, the discrete state-space model can be calculated based on the obtained order. Finally, the identified parameters are shown as follows: 


$$
\begin{aligned}
& A=\left[\begin{array}{cc}
-0.0005 & 0.0089 \\
-0.0087 & -0.0447
\end{array}\right] \quad B=\left[\begin{array}{cc}
-0.0150 & 0.1075 \\
-0.2702 & 0.5662
\end{array}\right] \\
& C=\left[\begin{array}{ll}
0.1083 & -0.6273 \\
0.0072 & -0.0065
\end{array}\right] \quad D=\left[\begin{array}{cc}
3.3420 & 2.9930 \\
-0.0978 & -0.0869
\end{array}\right]
\end{aligned}
$$

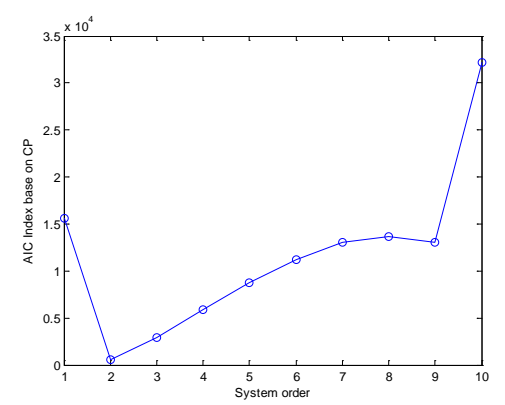

Fig. 3 AIC index based on CP method

The fitting curves have been calculated by using the identification data. The comparison between the model and plant outputs are shown in Fig. 4 and Fig. 5, respectively.

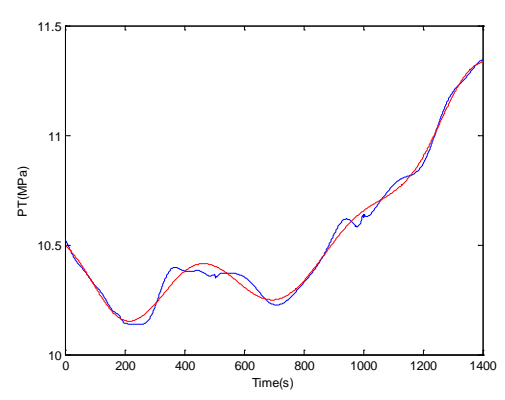

Fig. 4 Identification of throttle pressure

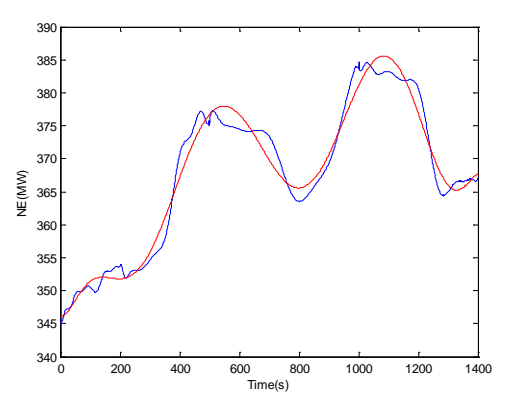

Fig. 5 Identification of output active power

The accuracy of predictive model can be determined by the prediction error, which can be written as following form.

$$
\operatorname{err}=\frac{1}{l} \sum_{j=1}^{l}\left[\sqrt{\frac{\sum_{i=1}^{N}\left(y h_{i j}-y_{i j}\right)^{2}}{\sum_{i=1}^{N}\left(y_{i j}\right)^{2}}}\right] \times 100 \%
$$

Where, $N$ is the data length, $l$ is the number of model output, $y h_{i j}$ and $y_{i j}$ are the values of simulation models and actual system respectively, where the subscript $i j$ denotes the $j$-th output value at the $i$-th moment. The prediction error of output active power, and the throttle pressure are $0.1356 \%$ and $0.0658 \%$, respectively. This fine error infers that the introduced method can provide an accurate identification. The simulation results show that the identification error is significantly fine.

\section{B. Dynamic simulations}

Under $60 \%$ rated capacity at stable point, the step disturbance responses of $\mu_{B}$ and $\mu_{T}$ is performed, respectively. The step response of $\mu_{B}$ is shown in Fig. 6.It can be seen that when $\mu_{T}$ is stable and fuel instruction $\mu_{B}$ is increased, the heat absorption of boiler heating surface evaporation must be increased too, while steam pressure is increased after a certain delay. Because of turbine tone opening is a constant during the simulation, the rise of steam pressure is limited by the increased steam flow spontaneously. When a new equilibrium has been obtained between the steam flow and combustion rate, steam pressure $P_{T}$ will tend to a new higher steady state. For the increase in steam flow, the output active power $N_{E}$ is also increased. 

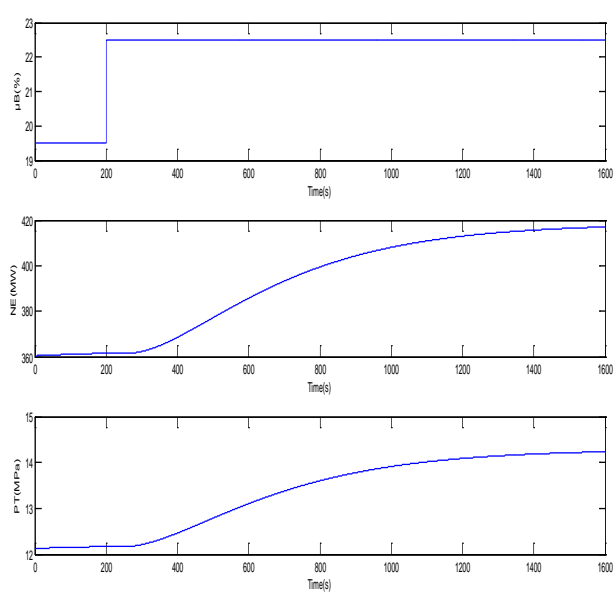

Fig. 6 The step response of $\mu_{B}$
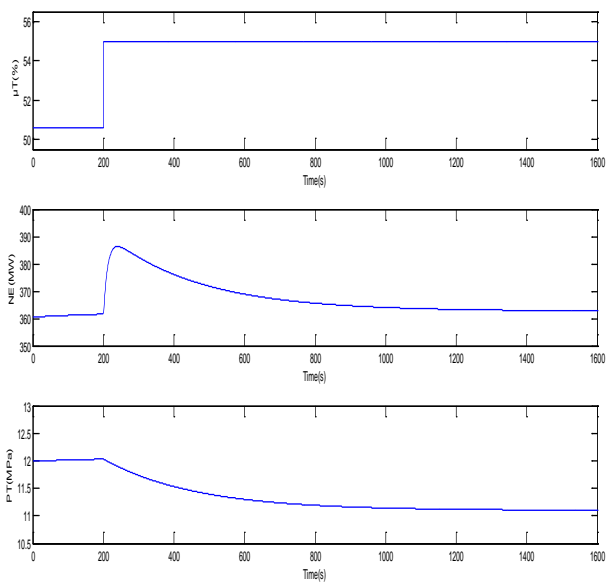

Fig. 7 The step response of $\mu_{T}$

The step response of $\mu_{T}$ is shown in Fig. 7. It can be seen that when $\mu_{B}$ is stable and fuel instruction $\mu_{T}$ is increased, steam flow must be increased immediately, and $P_{T}$ must be decreased at the same time. Because of $\mu_{B}$ is a constant during the simulation, the evaporation also remains the same. Because steam pressure is decreased, a part of the heat storage is released, which leads to the increase of steam flow, however, the process is temporary. In the end, the steam flow goes back to the initial value, throttle pressure $P_{T}$ will tend to a new lower steady state. Because steam flow is increased temporarily in the transition process, output active power $N_{E}$ is increased accordingly. Finally, output active power $N_{E}$ recovers the initial value.

\section{Conclusion}

In this paper, the SMI based on PCA is developed to identify parameters of the multivariable thermal process by choosing the appropriate field data. The data fitting of results and simulations show that the subspace based identification is an effective method of closed-loop identification for the multivariable objects, especially for the system with serious noise disturbance and large delay, such as the CCS of thermal power plant. This method can quickly and effectively identify the model of thermal power unit, which can better describe the identified system, and has a higher precision.

\section{Acknowledgement}

This work is supported by the National Key Basic Research Program of China (973 Program) under Grant No. 2012CB215203, and by the National Nature Science Foundation of China under Grant No. 61203043.

\section{References}

[1] FANG Fang, LIU Ji-zhen, TAN Wen. Multivariable IMC-PID Design in the Coordinated Control System for Fossil Unit Power Plants [J]. Power engineering, 2004,24 (3):36 0-365.

[2] D. Bauer, M. Deistler, W. Scherrer, Consistency and asymptotic normality of some subspace algorithms for systems without observed inputs, Automatica , 1999,35 (7):1243-1254.

[3] C.T. Chou, M. Verhaegen, Subspace algorithms for the identification of multivariable dynamic errors-in-variables models, Automatica, 1997,33 (10):1857-1869.

[4] M. Deistler, K.P. Scherrer, Consistency and relative efficiency of subspace methods, Automatica 31 (1995) 1865-1875. 
[5] M. Jansson, B. Wahlberg, On consistency of subspace methods for system identification, Automatica 34 (1998) 1507-1519.

[6] Verhaegen M. Application of a subspace model identification technique to identify LTI systems operating on closed-loop [J]. Automatica, 1993, 29(4):1027-1040.

[7] Ljung L, McKelvey T. Subspace identification from closed loop data [J].Signal Processing,1996,52:209-215.

[8] Van Overschee P, De Moor B. Closed-loop subspace system identification[A].Proceedings of the 36th Conference on Decision and Control,1997:1848-1853.

[9] Huang Biao, Ding Steven X, Qin S Joe. Closed-loop subspace identification-an orthogonal projection approach [J]. Journal of Process Control, 2005, 15: 53-66.

[10]Wang Jin, Qin S Joe, A new subspace identification approach based on principal component analysis. Journal of Process Control [J]. 2002(12): 841-855.

[11]B. Huang, S. X. Ding, S. J. Qin. Closed-loop subspace identification: An orthogonal projection approach. Journal of Process Control, 2005, 15(1): 53-66.

[12]F.P.de Mello. Boiler Models for System Dynamic Performance Studies. IEEE Transaction on Power Systems,1991,16(1).

[13] Cheres E. Small and medium size Drum Boiler Models Suitable for Long Term Dynamic Response [J]. IEEE Transaction on Energy Conversion,1900,5(4).

[14]H. Akaike, A new look at the statistical model identification, IEEE Trans. Auto. Cont. 19 (1974) 716-723.

[15]W.E. Larimore, System identification, reduced-order filtering and modeling via canonical variate analysis, In: H.S. Rao, T. Dorato (Eds.) Proc. 1983 American Control Conf. IEEE, New York.

[16]W.E. Larimore, Canonical variate analysis in identification, filtering and adaptive control. In Proc. 29th conference on decision and control. Honolulu, Hawaii, December (1990). 